\title{
MicroRNA-345 inhibits metastasis and epithelial-mesenchymal transition of gastric cancer by targeting FOXQ1
}

\author{
AIWEN FENG ${ }^{1}$, XIAOMING YUAN ${ }^{1}$ and XIANGWEI LI ${ }^{2}$ \\ Departments of ${ }^{1}$ Enterochirurgia and ${ }^{2}$ Gastroenterological Surgery, \\ Huaian First People's Hospital, Huaian, Jiangsu 223300, P.R. China
}

Received October 14, 2016; Accepted April 7, 2017

DOI: $10.3892 /$ or.2017.6001

\begin{abstract}
MicroRNAs (miRNAs) are a group of critical players in gastric cancer (GC). Among numerous cancerrelated miRNAs, the expression level and functional role of miR-345 in GC has not been investigated. This study showed that miR-345 expression was decreased in GC. Decreased expression level of miR-345 was associated with occurrence of lymph metastasis and advanced TNM stage of GC patients. Patients with low expression level of miR-345 had reduced overall survival (OS) and disease-free survival (DFS). In vitro experiments showed that miR-345 could inhibit the migration and invasion of GC cells. In vivo experiments showed that miR-345 knockdown could promote lung metastasis of GC cells in nude mice. miR-345 was found to prevent the metastasis by inhibiting epithelial-mesenchymal transition (EMT) of GC cells. Furthermore, FOXQ1 was confirmed to be the downstream target of miR-345 in GC cells. Forced expression of FOXQ1 could reverse the inhibitory effects of miR-345 on GC metastasis, while knockdown of FOXQ1 prevented the promoting effects of miR-345 knockdown on GC metastasis. In summary, this study demonstrates miR-345 is a promising biomarker and therapeutic target in GC.
\end{abstract}

\section{Introduction}

MicroRNAs (miRNAs), a group of noncoding RNAs with a length of approximately 14-22 nts, regulate genes expression by interacting with the 3'-UTR of mRNA of the targeted genes and preventing the translation or promoting the degradation of targeted mRNA (1). miRNAs have been found to be involved in various cellular functions including differentiation,

Correspondence to: Dr Xiaoming Yuan, Department of Enterochirurgia, Huaian First People's Hospital, 6 Beijing Xi Road, Huaiyin, Huaian, Jiangsu 223300, P.R. China

E-mail: yuanxiaoming1011@163.com

Key words: miR-345, gastric cancer, FOXQ1, metastasis, epithelialmesenchymal transition proliferation, apoptosis, and movements (2). The versatile roles of miRNAs have been implicated in different kinds of human diseases including cancers (3). miRNAs have been suggested as promising biomarkers and attractive therapeutic targets of human cancers (4). Moreover, studies of miRNAs in gastric cancer (GC) showed that miRNAs were critical regulators in the development and progression process in GC $(5,6)$. miRNAs were found to be active regulators for the growth and metastatic processes of GC (7). However, the expression status and biological functions of specific miRNA in GC remain to be investigated. Investigating the function and underlying mechanisms of miRNAs in GC is of great significance to identify novel biomarkers and treatment targets in GC.

miR-345 has been investigated in several human cancers. In non-small cell lung cancer, miR-345 was found to be downregulated and was associated with the patient prognosis (8). It was also found to induce the apoptosis of pancreatic cancer through both caspase-dependent and -independent pathways (9). In prostate cancer, miR-345 was found to suppress proliferation, migration and invasion of cancer cells by targeting Smad1 (10). However, miR-345 was found to be increased in locally advanced rectal cancer and was associated with unfavorable pathological response to preoperative chemoradiotherapy (11). This indicated miR-345 had different functional roles in human cancers. The expression and biological roles of miR-345 in GC have not been investigated.

In this study, miR-345 was found to be decreased in GC tissues and cell lines. Decreased expression of miR-345 was associated with adverse clinical features and unfavorable prognosis of GC patients. Functionally, miR-345 inhibited the metastasis of GC cells both in vitro and in vivo. miR-345 was found to inhibit epithelial-mesenchymal transition (EMT) of GC cells. Furthermore, FOXQ1, which is a well-known oncogenic protein and promoter of GC cell metastassis $(12,13)$, was confirmed to be the direct downstream target of miR-345 in GC. Inhibition of FOXQ1 expression was required for the inhibitory effects of miR-345 on the metastasis of GC cells.

\section{Materials and methods}

Cell culture and transfection. Gastric cancer cell lines including SGC-7901, AGS, MKN-45, MGC-803, and normal 
gastric epithelial cells GES-1 were obtained from the Cell Bank of Chinese Academy of Sciences (Shanghai, China) and American Type Culture Collection (ATCC, Rockville, MD, USA). Cells were maintained in DMEM medium (Gibco Co., Grand Island, NY, USA) with fetal bovine serum (10\%) (Gibco Co.). All cell cultures were kept at $37^{\circ} \mathrm{C}$ in a humidified incubator with $5 \% \mathrm{CO}_{2}$.

miR-345 mimic, miR-345 inhibitor, the control vector and negative control vector were obtained from Ruibo (Guangzhou, China). FOXQ1 vector and siRNA were purchased from GeneCopoeia (Guangzhou, China). The sequence of FOXQ1 siRNA was 5'-GCCCUGGCUCUCACAGCAA-3'. Cell transfections for GC cells were performed in 60-mm cell culture plates. The vectors including miR-345 mimic and inhibitor, and FOXQ1 vector and siRNA were transfected into GC cells with Lipofectamine 2000 (Invitrogen, Carlsbad, CA, USA). Generally, GC cells were $80-90 \%$ confluent before transfection. Lipofectamine reagent and the plasmids were diluted in Opti-MEM medium separately, and were mixed together at the ratio of 1:1. After incubating in room temperature for 5-10 min, the vector-lipid complex was added to GC cells. At 48-72 $\mathrm{h}$ after transfection, the transfected cells were collected for further assays.

Clinical tissues. Clinical specimens including gastric cancer tissues and adjacent non-tumor tissues were obtained from GC patients who underwent surgical resection in Department of Gastroenterological Surgery, Huaian First People's Hospital from 2005 to 2009. These clinical tissues samples were stored at $-80^{\circ} \mathrm{C}$ immediately after the surgical resection. All the tissues were pathologically confirmed as GC before subjecting to further experiments in this study. The protocol for experiments involving patient samples was approved by the Institutional Research Ethics Committee of Huaian First People's Hospital. The demographic and clinicopathological data of 85 patients are shown in Table I.

Quantitative real-time reverse transcription-PCR ( $q R T-P C R)$. RNeasy Mini kit (Qiagen) were used to extract the RNA from GC tissues and cells. Transcriptional First Strand cDNA Synthesis kit (Roche, Indianapolis, IN, USA) and SYBR Green PCR Master Mix (Applied Biosystems, Foster City, CA, USA) were used for reverse transcription reactions and real-time PCR. Primers for miR-345, U6, FOXQ1, E-caherin, $\mathrm{N}$-cadherin, vimentin, Zeb2, Snail1, and GAPDH were from Genecopoeia. The primer sequences are as follows: GAPDH: forward: 5'-AGGGCTGCTTTTAACTGGT-3', reverse: 5'-CCCCACTTGATTTTGGAGGGA-3'; FOXQ1: forward: 5'-AAGGATAAGGGTGACAGCAACAG-3', reverse: 5'-TTGCTGTGTAGGGACAGATTATGAC-3'; E-cadherin: forward: 5'-CACCTGGAGAGAGGCCATGT-3', reverse: 5'-TGGGAAACATGAGCAGCTCT-3'; N-cadherin: forward: 5'-ATGTGCCGGATAGCGGGAGC-3', reverse: 5'-TACA CCGTGCCGTCCTCGTC-3'; vimentin: forward: 5'-CTTGA ACGGAAAGTGGAATCCT-3', reverse: 5'-GTCAGGCTT GGAAACGTCC-3'; Snail1: forward: 5'-AAGATGCACATC CGAAGCCA-3', reverse: 5'-CTCTTGGTGCTTGTGGAG CA-3'; Zeb2: forward: 5'-TCTGAAGATGAAGAAGGCTG-3', reverse: 5'-AGTGAATGAGCCTCAGGTAA-3'. U6 was the internal control for miR-345 while GAPDH was the internal
Table I. Clinical association analysis of miR-345 expression in gastric cancer.

\begin{tabular}{|c|c|c|c|c|}
\hline \multirow[b]{2}{*}{ Clinical features } & \multirow{2}{*}{$\begin{array}{c}\text { No. of } \\
\text { patients }\end{array}$} & \multicolumn{2}{|c|}{ miR-345 level } & \multirow[b]{2}{*}{ P-value } \\
\hline & & Low & High & \\
\hline Age (years) & & & & 0.125 \\
\hline$<50$ & 36 & 14 & 22 & \\
\hline$\geq 50$ & 49 & 28 & 21 & \\
\hline Sex & & & & 0.380 \\
\hline Male & 51 & 23 & 28 & \\
\hline Female & 34 & 19 & 15 & \\
\hline Size & & & & 0.274 \\
\hline$<5 \mathrm{~cm}$ & 36 & 15 & 21 & \\
\hline$\geq 5 \mathrm{~cm}$ & 49 & 27 & 22 & \\
\hline Lymph node metastasis & & & & $<0.001$ \\
\hline No & 35 & 6 & 29 & \\
\hline Yes & 50 & 36 & 14 & \\
\hline TNM stage & & & & 0.002 \\
\hline I-II & 49 & 17 & 32 & \\
\hline III-IV & 36 & 25 & 11 & \\
\hline
\end{tabular}

P-value represents Chi-square test for the correlation between miR-345 level and clinical features. $\mathrm{P}<0.05$ indicates miR-345 is closely associated with this clinical feature.

control for E-cadherin, N-cadherin, vimentin, Zeb2, Snail1 and FOXQ1.

Western blotting. Total protein lysates extracted from GC cells were subjected to BCA protein assay kit (Pierce, Rockford, IL, USA) for protein concentration. Total protein lysates (20-30 $\mu \mathrm{g})$ extracted from samples were separated in $10 \%$ SDS-PAGE gels and were transferred to polyvinylidene fluoride membrane. The membrane was blocked with 5\% milk/TBST (0.1\% Tween), followed by incubating with following primary antibodies including FOXQ1 (1:1000, Cell Signaling Technology, Danvers, MA, USA), E-acdherin (1:1000, Cell Signaling Technology), N-cadherin (1:500, Cell Signaling Technology), FOXQ1 (1:500; Santa Cruz Biotechnology Inc., Santa Cruz, CA, USA), SNAIL1 (1:1000, Cell Signaling Technology), ZEB2 (1:800, Cell Signaling Technology) and GAPDH (1:2000, Santa Cruz Biotechnology Inc.) were incubated with the membrane overnight at $4^{\circ} \mathrm{C}$. After incubating with secondary antibodies (1:3000, Santa Cruz Biotechnology Inc.) for $1 \mathrm{~h}$ at room temperature, the protein signals were visualized using ECL reagents (Amersham Biosciences Corp., Piscataway, NJ, USA).

Transwell assays. The migration and invasion ability of GC cells were investigated by Transwell assays. For migration ability, Transwell chambers (Corning Inc., Corning, NY, USA) without matriel coating were used. For invasion assay, Transwell chambers coated with matrigel (Corning Inc.) were used. Generally, GC suspended in basal DMEM were seeded into the upper 

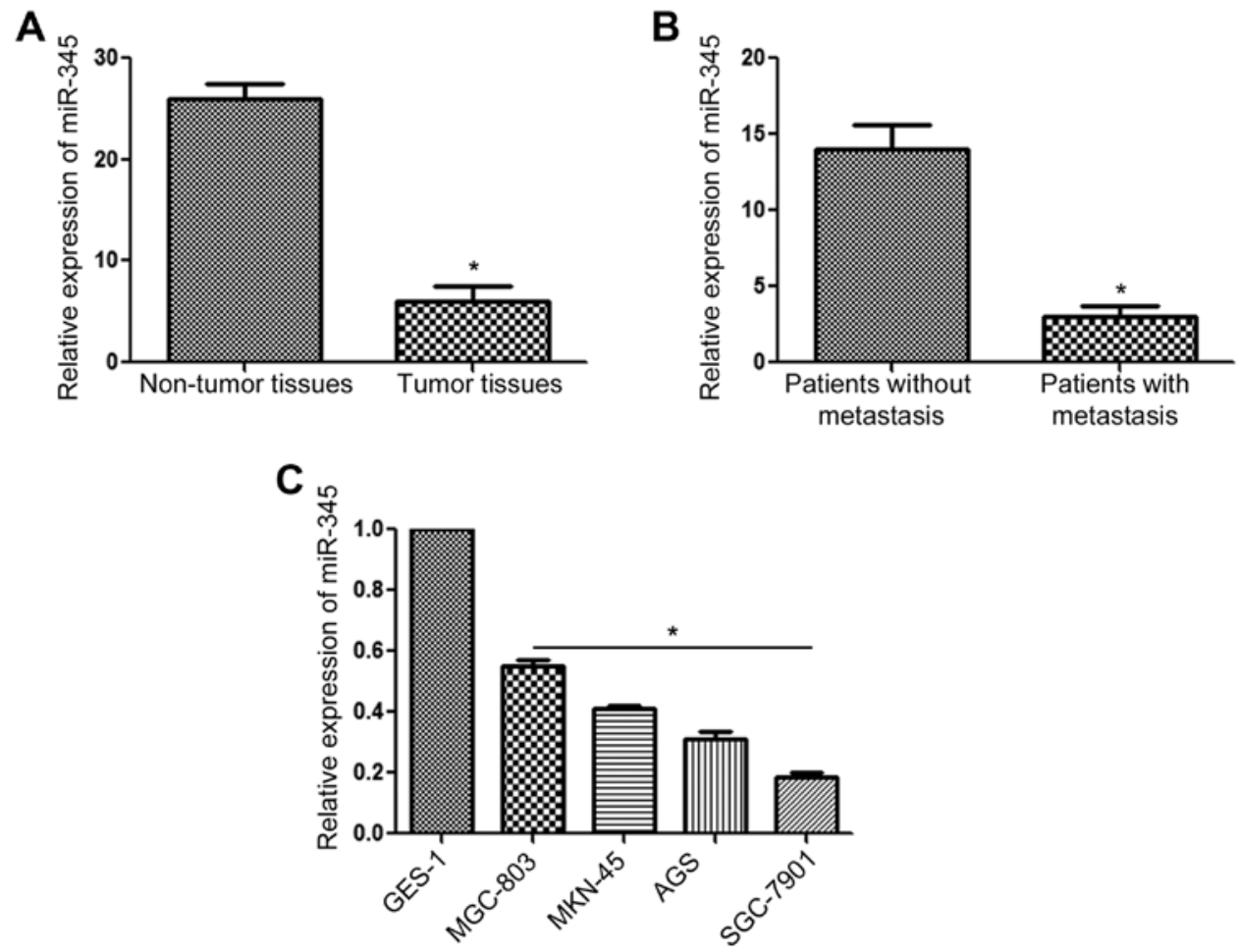

Figure 1. The expression of miR-345 in gastric cancer. (A) The expression of miR-345 was compared between gastric cancer tissues and matched adjacent normal tissues. (B) The expression of miR-345 was compared between patients with metastasis and those without metastasis. (C) The expression of miR-345 was compared between four gastric cancer cell lines and the GES-1 cells. ${ }^{*} \mathrm{P}<0.05$. $\mathrm{n}=3$. The error bars are SEM.

A

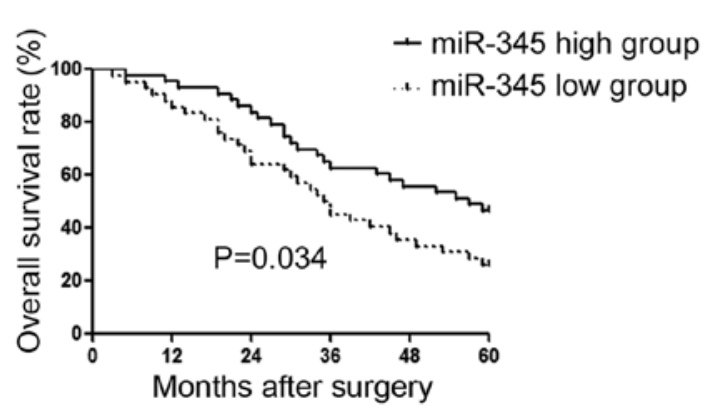

B

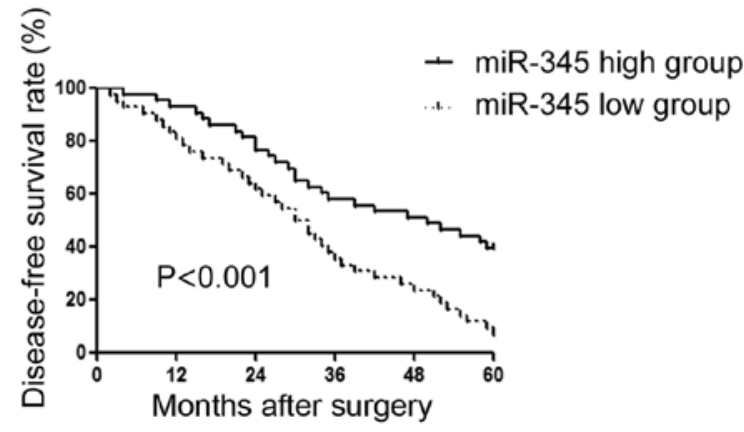

Figure 2. The prognostic value of miR-345 in gastric cancer. Kaplan-Meier plots were used to compare the (A) Overall survival rate and (B) disease-free survival rate between patients with high level of miR-345 and those with low miR-345 level.

chamber and the lower chambers contained $600 \mu 1 \mathrm{DMEM}$ with $20 \%$ FBS. After 24-48 h, GC cells migrated or invaded through the membranes and stayed on the lower surface were stained with crystal violet. Cell number for the migrated or invaded cells was counted under a microscope.

Luciferase assay. Wild-type 3'-UTR sequence of FOXQ1 containing target site of miR-345 or the mutated 3'-UTR sequence of FOXQ1 was incorporated into the pGL3 control vector (Promega, Madison, WI, USA), to get the wild-type FOXQ1-3'UTR or mutant FOXQ1-3'UTR, respectively. GC cells were seeded into 24-well plates the day before transfection, and then cells were cotransfected with wild-type or mutant 3'-UTR of FOXQ1 along with miR-345 mimic or inhibitor. At $48 \mathrm{~h}$ after co-transfection, the luciferase activity for the wild-type or mutant FOXQ1 3'-UTR was measured by a single luciferase reporter assay (Promega).

In vivo metastasis assay. To evaluate the metastatic ability of GC cells in vivo, tail vein injection model was performed. GC cells transfected with negative control vector or miR-345 inhibitor were injected through tail vein into nude mice. The lungs of nude mice were subjected to hematoxylin and eosin (H\&E) staining for lung metastasis of GC cells after 5 weeks. All animal experiments were approved by the Animal Care Committee of Huaian First People's Hospital.

Statistical analysis. All data are presented as the mean \pm standard error (SE) and were analyzed using the Graphpad. Student't test, Chi-square, Correlation analysis, and Kaplan-Meier 

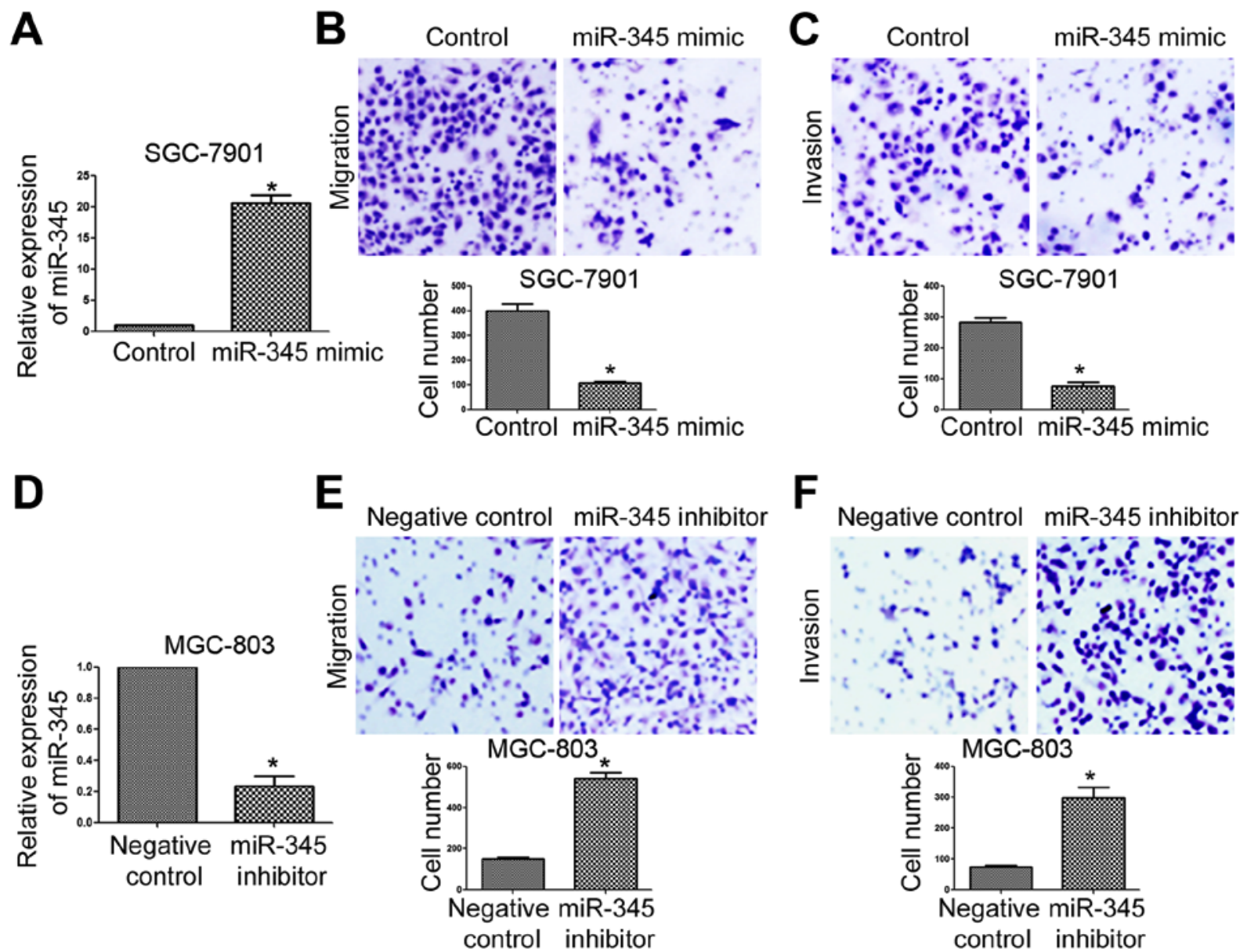

Figure 3. miR-345 inhibits in vitro migration and invasion of GC cells. (A) miR-345 mimic significantly increased the expression of miR-345 in SGC-7901 cells. (B) The migration and (C) invasion of SGC-7901 cells was significantly decreased after miR-345 overexpression. (D) miR-345 inhibitor significantly decreased the expression of miR-345 in MGC-803 cells. (E) The migration and (F) invasion of MGC-803 cells was significantly increased after miR-345 inhibition. ${ }^{*} \mathrm{P}<0.05$. $\mathrm{n}=3$. The error bars are SEM.

analysis were performed in this study for statistical analysis. $\mathrm{P}<0.05$ was considered to be statistically significant.

\section{Results}

miR-345 expression is decreased in GC. To evaluate the expression level of miR-345 in GC, we collected RNA from the GC tissues and adjacent non-tumor tissues and performed qRT-PCR for the extracted RNA. qRT-PCR showed that compared with adjacent normal tissues, the expression level of miR-345 in GC tissues was decreased significantly (Fig. 1A, $\mathrm{P}<0.05$ ). Then, we divided the enrolled patients into two groups: patients with metastasis and those without metastasis. Compared with those without metastasis, patients with metastasis showed significantly decreased level of miR-345 (Fig. 1B, P<0.05). Furthermore, the expression of miR-345 in GC cell lines and GES-1 cells. Compared with GES-1 cells, miR-345 in all four GC cell lines was significantly decreased (Fig. 1C, $\mathrm{P}<0.05$ ).

Decreased miR-345 expression level is correlated with poor clinicopathological features and prognosis of GC patients. After confirming the decreased expression of miR-345 in GC, we evaluated the clinical significance of the decreased expression level of miR-345. Clinical association analysis (Table I) showed that decreased expression level of miR-345 was associated with the occurrence of lymphatic metastasis $(\mathrm{P}<0.001)$ and advanced TNM stage $(\mathrm{P}=0.002)$. Kaplan-Meier analysis further showed patients with relatively lower level of miR-345 had significantly decreased overall survival (Fig. 2A, $\mathrm{P}=0.034$ ) and disease-free survival (Fig. 2B, $\mathrm{P}<0.001$ ).

miR-345 inhibits the migration and invasion of GC cells both in vitro and in vivo. As suggested in Fig. 1C, miR-345 level was the lowest in SGC-7901 cells and the highest in MGC-803 cells. Therefore, we chose SGC-7901 cells for overexpression assay and MGC-803 cells for knockdown assay. As shown in Fig. 3A, overexpression of miR-345 significantly increased the level of miR-345 in SGC-7901 cells (Fig. 3A, $\mathrm{P}<0.05$ ). Functionally, miR-345 overexpression significantly inhibited the migration (Fig. 3B, $\mathrm{P}<0.05$ ) and invasion (Fig. $3 \mathrm{C}, \mathrm{P}<0.05$ ) of SGC-7901 cells. On the contrary, miR-345 inhibitor significantly reduced miR-345 level in MGC-803 cells (Fig. 3D, $\mathrm{P}<0.05$ ), and increased the migration (Fig. $3 \mathrm{E}, \mathrm{P}<0.05$ ) and invasion (Fig. 3F, P<0.05) of MGC-803 cells.

miR-345 knockdown potentiates the lung metastasis of GC cells in nude mice. To further confirm the in vitro effects of 


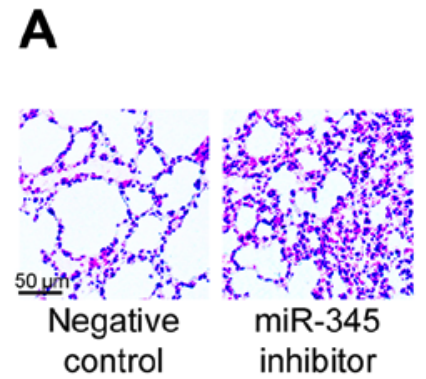

MGC-803

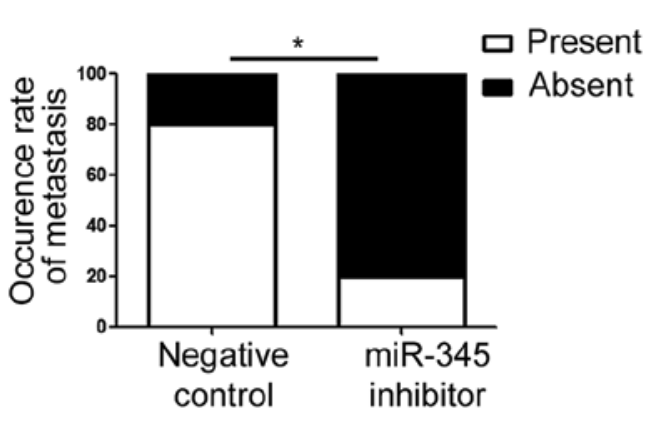

Figure 4. miR-345 inhibits the lung metastasis of GC cells in nude mice. (A) Hematoxylin and eosin (H\&E) staining and the rate of lung metastasis were compared between MGC- 803 cells transfected with miR-345 inhibitor and those transfected with negative control vector. (B) The number of metastatic nodules were compared between MGC-803 cells transfected with miR-345 inhibitor and those transfected with negative control vector. ${ }^{*} \mathrm{P}<0.05$. $\mathrm{n}=3$. The error bars are SEM.
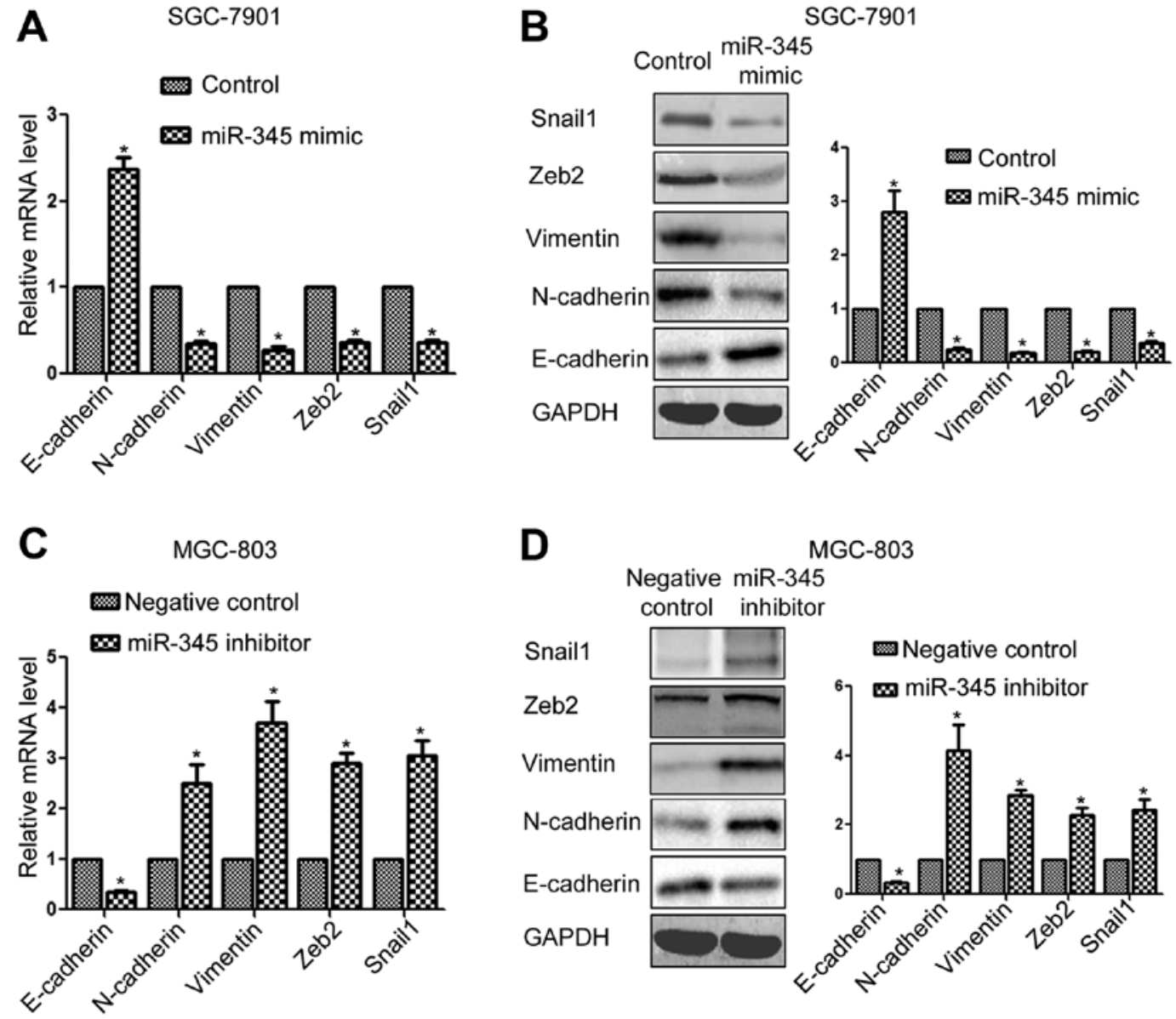

Figure 5. miR-345 inhibits the EMT of GC cells. (A) miR-345 overexpression significantly increased the mRNA level of E-cadherin while decreased the mRNA level of N-cadherin, vimentin, Zeb2 and Snail1 in SGC-7901 cells. (B) miR-345 overexpression significantly increased the expression of E-cadherin while decreased the expression of N-cadherin, vimentin, Zeb2 and Snail1 in SGC-7901 cells. (C) miR-345 downregulation significantly decreased the mRNA level of E-cadherin while increased the mRNA level of N-cadherin, vimentin, Zeb2, Snail1 in MGC-803 cells. (D) miR-345 downregulation significantly decreased the expression of E-cadherin while increased the expression of N-cadherin, vimentin, Zeb2, Snaill in MGC-803 cells. "P<0.05. $\mathrm{n}=3$. The error bars are SEM.

miR-345 on the metastatic behavior of GC cells, we performed tail vein injection to evaluate whether miR-345 could inhibit the metatasis of GC cells in nude mice. As shown in Fig. 4A, knockdown of miR-345 in MGC-803 cells significantly increased the rate of lung metastasis in nude mice $(\mathrm{P}<0.05)$. Further analysis of the lung of nude mice showed that miR-345 knockdown significantly increased the number of metastatic nodules in lungs of nude mice (Fig. 4B, $\mathrm{P}<0.05$ ).
miR-345 inhibits the epithelial-mesenchymal transition (EMT) of GC cells. EMT is a critical mechanism for the metastasis of cancer cells (14). We investigated whether miR-345 could inhibit the metastasis of GC cells by inhibiting EMT phenotype. As shown in Fig. 5A, overexpression of miR-345 significantly increased the expression of E-cadherin and decreased $\mathrm{N}$-cadherin and vimentin expression $(\mathrm{P}<0.05$, Fig. 5A). Of note, the transcriptional regulators of EMT 


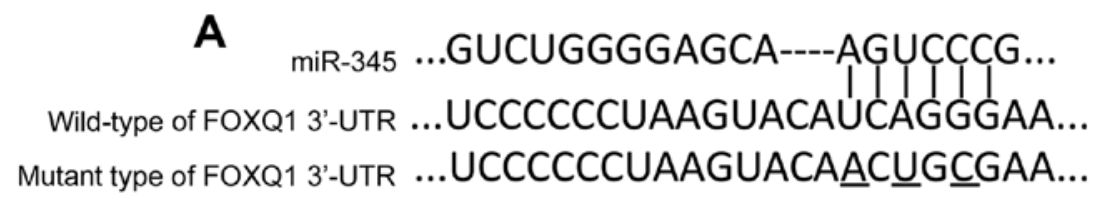

B

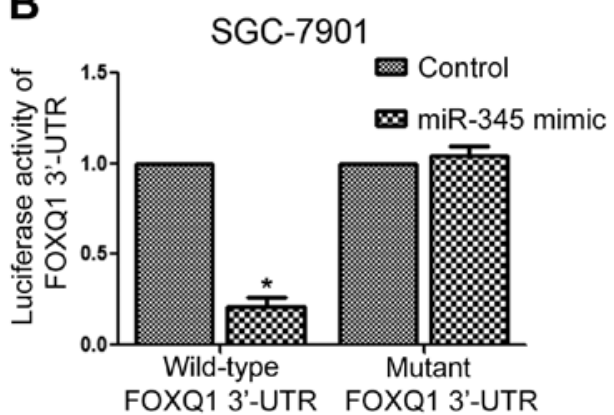

C

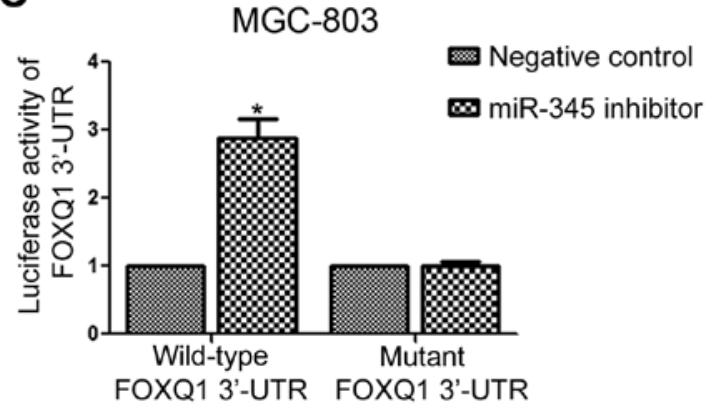

Figure 6. miR-345 inhibits the lucifease acitivity of FOXQ1 3'-UTR in GC cells. (A) 3'-UTR of FOXQ1 has the complementary sequence for miR-345 binding. (B) miR-345 overexpression significantly reduced while (C) miR-345 inhibition significantly increased the luciferase activity of wild-type but not mutant 3'-UTR of FOXQ1. "P<0.05. $\mathrm{n}=3$. The error bars are SEM.
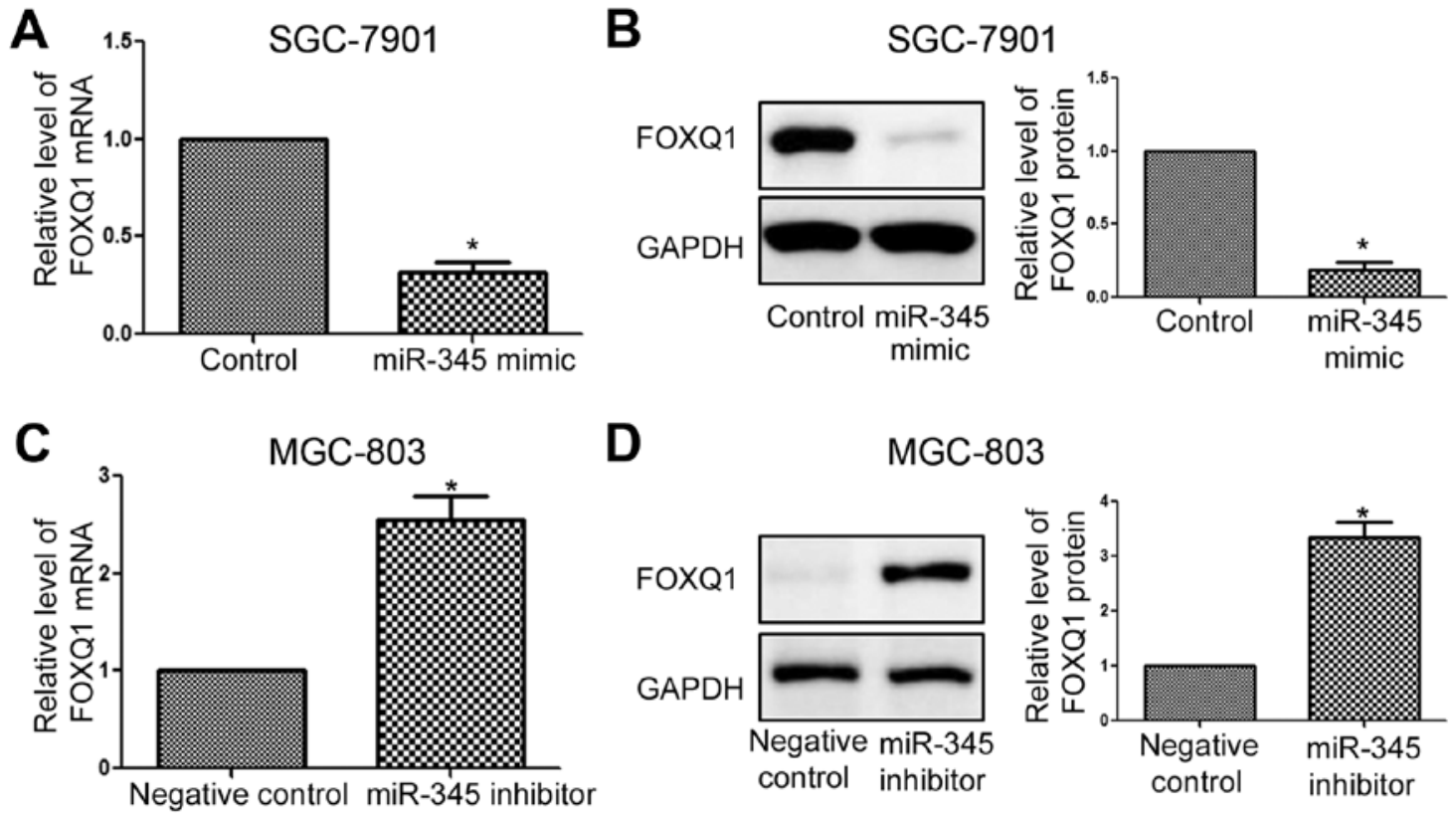

Figure 7. miR-345 inhibits the expression of FOXQ1 in GC cells. miR-345 overexpression significantly reduced the (A) mRNA and (B) protein level of FOXQ1 in SGC-7901 cells. miR-187 knockdown increased FOXQ1 (C) mRNA and (D) protein level in MGC-803 cells. * $\mathrm{P}<0.05$. $\mathrm{n}=3$. The error bars are SEM.

including Snaill and Zeb2 was also found to be decreased after miR-345 overexpression ( $\mathrm{P}<0.05$, Fig. 5A). On the other hand, miR-345 inhibition significantly reduced E-cadherin expression and increased $\mathrm{N}$-cadherin and vimentin expression, and increased the expression of Snaill and Zeb2 ( $\mathrm{P}<0.05$, Fig. 5B).

FOXQ1 is the downstream target of miR-345 in GC cells. The significant influence of miR-345 on the metastasis of GC cells prompted us to investigate the underlying mechanism for its functional influence. The data from Targetscan showed that FOXQ1 had complementary binding sites for miR-345 (Fig. 6A). Then, we performed luciferase assay and found that overexpression of miR-345 significantly inhibited (Fig. 6B, $\mathrm{P}<0.05)$ while inhibition of miR-187 significantly increased
(Fig. 6C, P<0.05) the luciferase activity of wild-type 3'-UTR of FOXQ1. Altering miR-345 expression did not affect the luciferase activity of mutant 3'-UTR of FOXQ1 in GC cells (Fig. 6B and C).

Furthermore, we performed qRT-PCR and western blotting to confirm that FOXQ1 was the downstream target of miR-345. We found that overexpression of miR-345 significantly decreased the mRNA level (Fig. 7A, $\mathrm{P}<0.05)$ and protein (Fig. 7B, $\mathrm{P}<0.05$ ) level of FOXQ1. On the other hand, inhibition of miR-345 significantly increased the mRNA (Fig. 7C, $\mathrm{P}<0.05$ ) and protein (Fig. 7D, $\mathrm{P}<0.05$ ) level of FOXQ1.

FOXQ1 mediates the biological functions of miR-187 in GC cells. Since we confirmed FOXQ1 was the direct downstream 


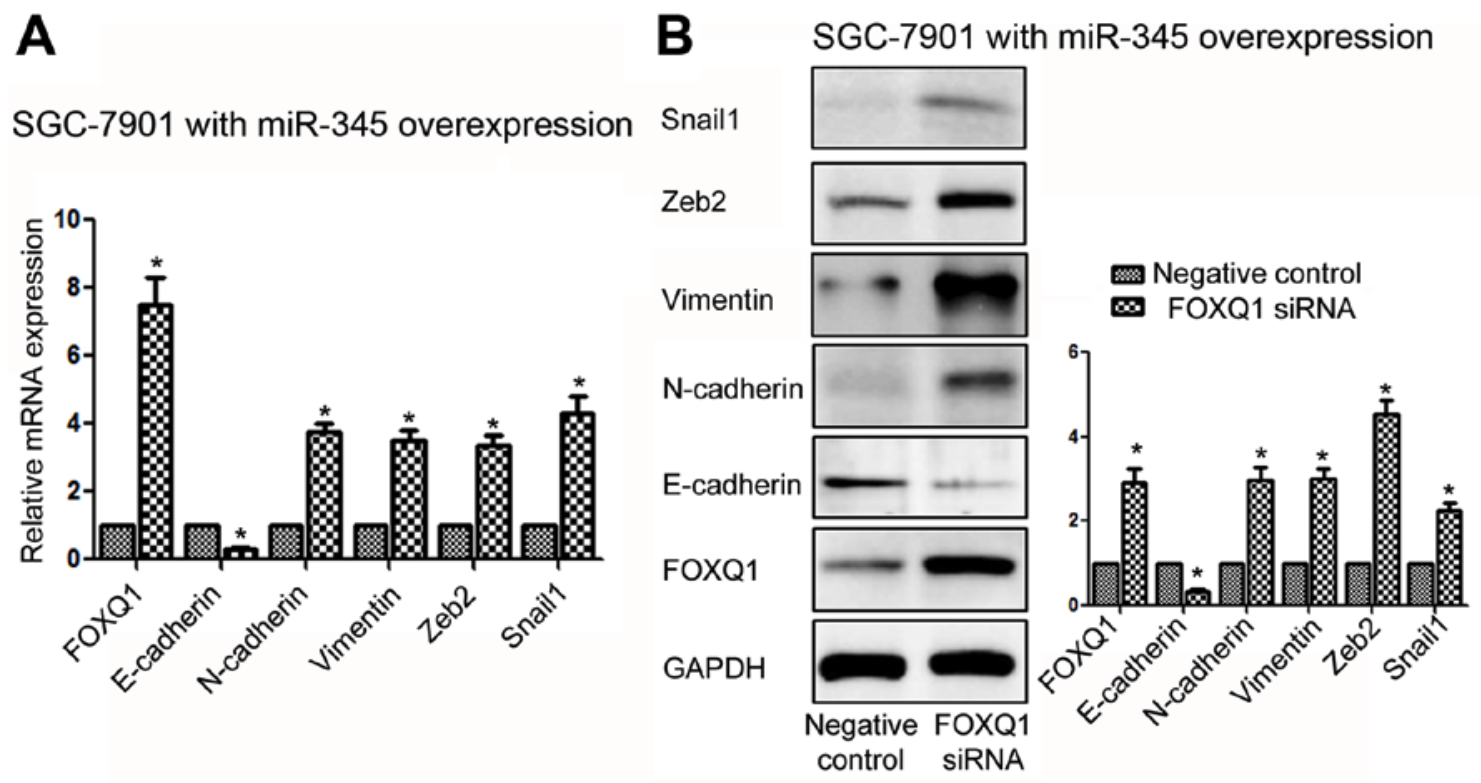

C SGC-7901 with miR-345 overexpression
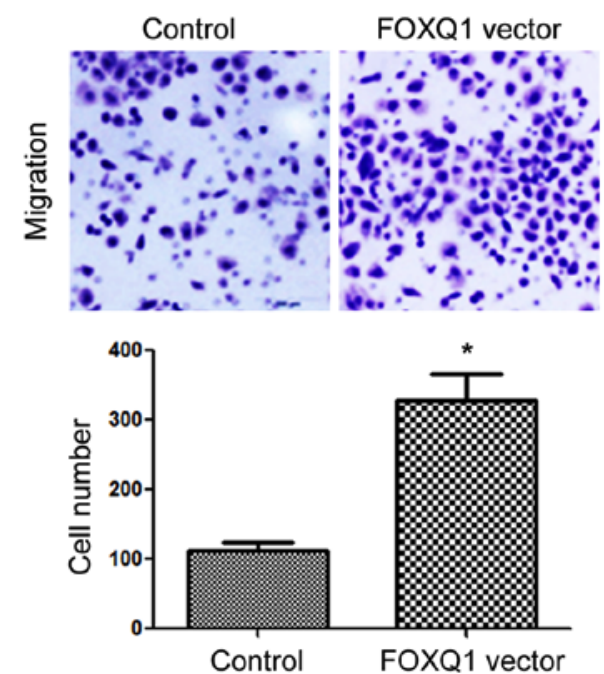

D

SGC-7901 with miR-345 overexpression
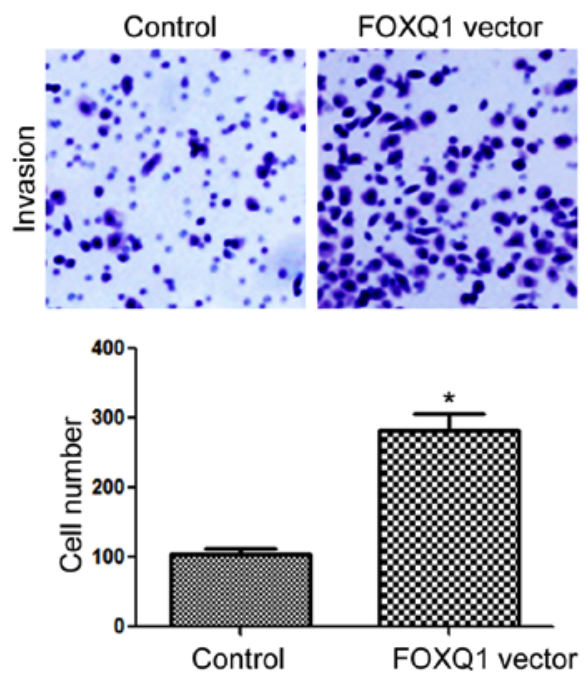

Figure 8. FOXQ1 overexpression abrogates the inhibitory effects of miR-345 overexpression on EMT, migration and invasion of SGC-7901 cells. (A) FOXQ1 expression vector significantly increased mRNA level of FOXQ1 in SGC-7901 cells transfected with miR-345 mimic, and subsequently led to decreased mRNA level of E-cadherin and increased mRNA level of N-cadherin, vimentin, Zeb2 and Snaill. (B) FOXQ1 expression vector significantly increased the expression of FOXQ1 in SGC-7901 cells transfected with miR-345 mimic, and subsequently led to decreased expression of E-cadherin and increased expression of N-cadherin, vimentin, Zeb2 and Snail1. Overexpression of FOXQ1 abrogated the inhibiting effects of miR-345 overexpression on (C) migration and (D) invasion. ${ }^{*} \mathrm{P}<0.05$. $\mathrm{n}=3$. The error bars are SEM.

target of miR-345 in GC cells, we examined whether targeting FOXQ1 was required for the inhibitory effects of miR-345 on GC cell metastasis and EMT. We overexpressed FOXQ1 in SGC-7901 cells transfected with miR-345 mimic, and FOXQ1 vector significantly increased the expression of FOXQ1 in SGC-7901 cells transfected with miR-345 mimic (Fig. 8A, $\mathrm{P}<0.05)$. Overexpressing FOXQ1 reversed the inhibitory effects of miR-345 overexpression on EMT as suggested by the increased expression of E-cadherin, and decreased expression of $\mathrm{N}$-cadherin, vimentin, Zeb2 and Snail1 (Fig. 8A, $\mathrm{P}<0.05$ ). Functionally, overexpressing FOXQ1 in SGC-7901 cells transfected with miR-345 mimic reduced the inhibitory effects of miR-345 overexpression on the metastasis of GC cells, as suggested by increased migration $($ Fig. $8 \mathrm{~B}, \mathrm{P}<0.05)$ and invasion (Fig. 8C, $\mathrm{P}<0.05$ ) of SGC-7901 cells transfected with miR-345 mimic. On the other hand, FOXQ1 siRNA significantly decreased the expression of FOXQ1 in MGC-803 cells with miR-345 inhibitor (Fig. 9A, $\mathrm{P}<0.05$ ). Inhibition of FOXQ1 prevented the promoting effects of miR-345 knockdown on the EMT phenotype (Fig. 9A, $\mathrm{P}<0.05$ ), migration (Fig. 9B, $\mathrm{P}<0.05$ ) and invasion (Fig. 9C, $\mathrm{P}<0.01$ ) of MGC-803 cells.

\section{Discussion}

Gastric cancer (GC) which is the second cause of cancerrelated mortality worldwide has a 5-year overall survival 
A

MGC-803 with miR-345 knockdown

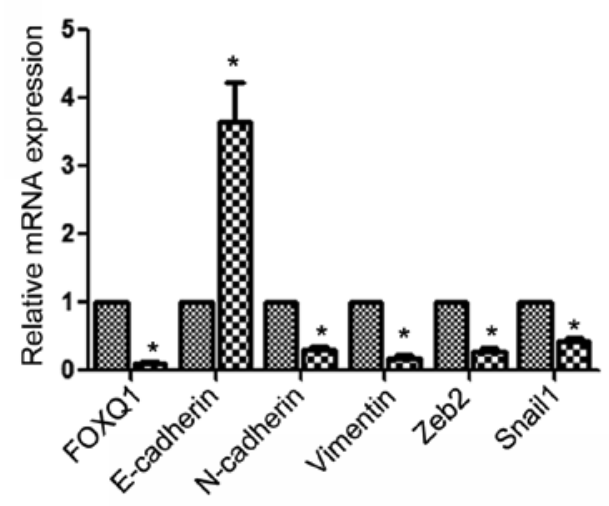

\section{C}
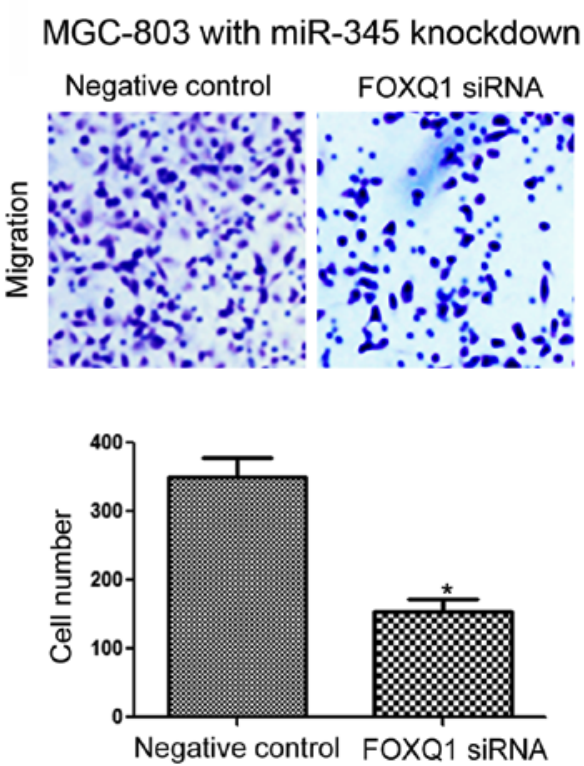

B

Snail1

Zeb2

Vimentin

$\mathrm{N}$-cadherin

E-cadherin

FOXQ1

GAPDH

Negative FOXQ1

control SiRNA
MGC-803 with miR-345 knockdown

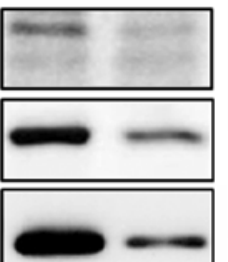

Negative control * FOXQ1 siRNA

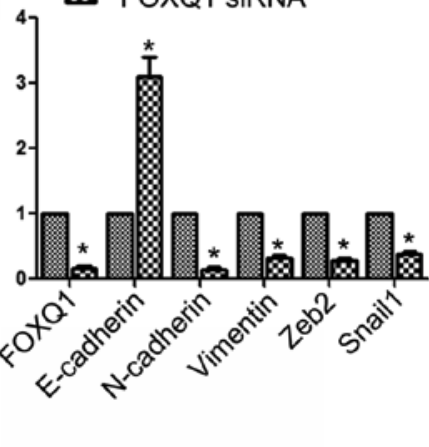

D MGC-803 with miR-345 knockdown
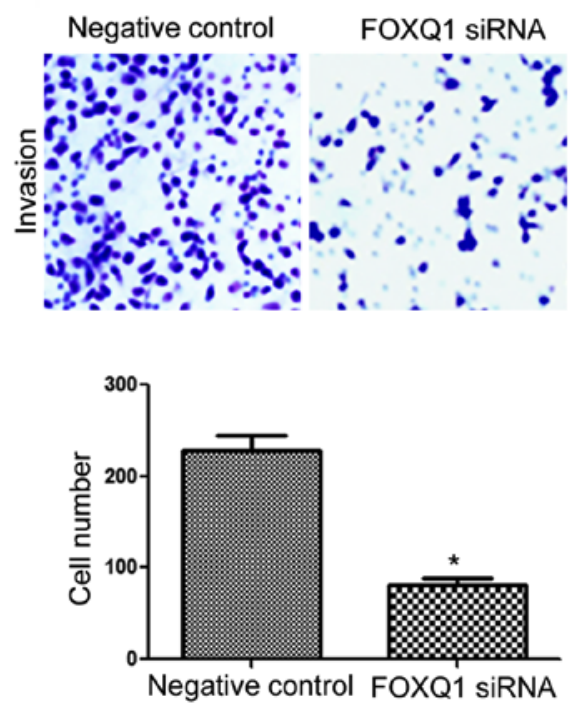

Figure 9. Inhibition of FOXQ1 reverses the promoting effects of miR-345 kncokdown on EMT, migration and invasion of MGC-803 cells. (A) FOXQ1 siRNA significantly decreased mRNA level of FOXQ1 in MGC-7901 cells transfected with miR-345 inhibitor, and subsequently led to increased mRNA level of E-cadherin and decreased mRNA level of N-cadherin, vimentin, Zeb2 and Snail1. (B) FOXQ1 siRNA significantly decreased the expression of FOXQ1 in MGC-7901 cells transfected with miR-345 inhibitor, and subsequently led to increased expression of E-cadherin and decreased expression of N-cadherin, vimentin, Zeb2 and Snail1. FOXQ1 knockdown abrogated the promoting effects of miR-345 knockdown on (C) migration and (D) invasion. ${ }^{*} \mathrm{P}<0.05$. $\mathrm{n}=3$. The error bars are SEM.

$<30 \%(15,16)$. Occurrence of systemic metastasis is the most important reason for the poor prognosis of GC patients in advanced stage $(17,18)$. Investigating the molecular mechanisms underlying the metastasis of GC will be critical for improving the prognosis of $\mathrm{GC}$ patients.

miRNAs have been confirmed as important regulators of cancer metastasis $(19,20)$. Among numerous miRNAs, miR-187 has been confirmed as a critical regulator of human cancers including non-small cell lung cancer (8), pancreatic cancer (9), prostate cancer (10) and rectal cancer (11). In this study, we found that miR-345 expression was significantly decreased in GC tissues and cell lines. Decreased expression of miR-345 was associated with adverse clinicopathological features and poor prognosis of GC patients. Functionally, miR-345 was found to inhibit the metastasis of GC cells both in vitro and in vivo. Moreover, we further explored whether miR-345 inhibited the metastasis of GC cells through inhibiting EMT phenotype (as suggested by increased E-cadherin and decreased $\mathrm{N}$-cadherin and vimentin). The data of western blotting (WB) showed that miR-345 could inhibit the epithelial-mesenchymal transition of GC cells.

FOXQ1 has been confirmed as an important player in cancer metastasis (21). Study of gastric cancer showed that FOXQ1 promoted the metastasis and EMT of GC cells by upregulating Snail1 (22). Increased FOXQ1 expression has been regarded as a prognostic indicator of GC patients (12). In 
this study, our data from luciferase assay, qRT-PCR and WB solidly confirmed that FOXQ1 was the direct downstream target of miR-345 in GC cells. Importantly, rescue experiments and functional assays demonstrated that targeting FOXQ1 was required for the inhibitory effects of miR-345 on the metastasis and EMT of GC cells. These data indicated that miR-345 inhibited the metastasis and EMT of GC cells by targeting FOXQ1.

In summary, miR-345 was decreased in GC tissues and cells. Decreased level of miR-187 was associated with the lymphatic metastasis and advanced TNM stage and poor prognosis of GC patients. miR-345 was able to inhibit the metastasis of GC cells both in vitro and in vivo. miR-345 inhibited the EMT of GC cells. Furthermore, we confirmed that FOXQ1 was identified as the downstream target of miR-345 in GC cells, and was required for the functional influence of miR-345 on metastasis and EMT of GC cells. The above demonstrate miR-345 is an attractive biomarker and therapeutic target for GC patients.

\section{References}

1. Ha M and Kim VN: Regulation of microRNA biogenesis. Nat Rev Mol Cell Biol 15: 509-524, 2014.

2. Alvarez-Garcia I and Miska EA: MicroRNA functions in animal development and human disease. Development 132: 4653-4662, 2005.

3. Gregory RI and Shiekhattar R: MicroRNA biogenesis and cancer. Cancer Res 65: 3509-3512, 2005.

4. Bartels CL and Tsongalis GJ: MicroRNAs: Novel biomarkers for human cancer. Clin Chem 55: 623-631, 2009.

5. Ueda T, Volinia S, Okumura H, Shimizu M, Taccioli C, Rossi S, Alder H, Liu CG, Oue N, Yasui W, et al: Relation between microRNA expression and progression and prognosis of gastric cancer: A microRNA expression analysis. Lancet Oncol 11: 136-146, 2010.

6. Volinia S, Calin GA, Liu C-G, Ambs S, Cimmino A, Petrocca F, Visone R, Iorio M, Roldo C, Ferracin M, et al: A microRNA expression signature of human solid tumors defines cancer gene targets. Proc Natl Acad Sci USA 103: 2257-2261, 2006.

7. Wu WK, Lee CW, Cho CH, Fan D, Wu K, Yu J and Sung JJ: MicroRNA dysregulation in gastric cancer: A new player enters the game. Oncogene 29: 5761-5771, 2010.
8. Chen L, Li X and Chen X: Prognostic significance of tissue miR-345 downregulation in non-small cell lung cancer. Int J Clin Exp Med 8: 20971-20976, 2015.

9. Srivastava SK, Bhardwaj A, Arora S, Tyagi N, Singh S, Andrews J, McClellan S, Wang B and Singh AP: MicroRNA-345 induces apoptosis in pancreatic cancer cells through potentiation of caspase-dependent and -independent pathways. Br J Cancer 113: 660-668, 2015

10. Chen QG, Zhou W, Han T, Du SQ, Li ZH, Zhang Z, Shan GY and Kong CZ: MiR-345 suppresses proliferation, migration and invasion by targeting Smad1 in human prostate cancer. J Cancer Res Clin Oncol 142: 213-224, 2016.

11. Yu J, Li N, Wang X, Ren H, Wang W, Wang S, Song Y, Liu Y, Li Y, Zhou X, et al: Circulating serum microRNA-345 correlates with unfavorable pathological response to preoperative chemoradiotherapy in locally advanced rectal cancer. Oncotarget 7: 64233-64243, 2016.

12. Liang S-H, Yan X-Z, Wang B-L, Jin HF, Yao LP, Li YN, Chen M, Nie YZ, Wang X, Guo XG, et al: Increased expression of FOXQ1 is a prognostic marker for patients with gastric cancer. Tumour Biol 34: 2605-2609, 2013.

13. Zhang J, Liu Y, Zhang J, Cui X, Li G, Wang J, Ren H and Zhang Y: FOXQ1 promotes gastric cancer metastasis through upregulation of Snail. Oncol Rep 35: 3607-3613, 2016.

14. Yilmaz M and Christofori G: EMT, the cytoskeleton, and cancer cell invasion. Cancer Metastasis Rev 28: 15-33, 2009.

15. Carcas LP: Gastric cancer review. J Carcinog 13: 14, 2014.

16. Yasui W, Oue N, Aung PP, Matsumura S, Shutoh M and Nakayama H: Molecular-pathological prognostic factors of gastric cancer: A review. Gastric Cancer 8: 86-94, 2005.

17. Tsugane $S$ and Sasazuki S: Diet and the risk of gastric cancer: Review of epidemiological evidence. Gastric Cancer 10: 75-83, 2007.

18. Crew KD and Neugut AI: Epidemiology of gastric cancer. World J Gastroenterol 12: 354-362, 2006

19. Hampton T: MicroRNA and Metastasis. JAMA 298: 1998, 2007. http://jamanetwork.com/journals/jama/article-abstract/209330.

20. Yates LA, Norbury CJ and Gilbert RJ: The long and short of microRNA. Cell 153: 516-519, 2013.

21. Qiao Y, Jiang X, Lee ST, Karuturi RK, Hooi SC and Yu Q: FOXQ1 regulates epithelial-mesenchymal transition in human cancers. Cancer Res 71: 3076-3086, 2011.

22. Xiang X-J, Deng J, Liu Y-W, Wan LY, Feng M, Chen J and Xiong JP: MiR-1271 inhibits cell proliferation, invasion and EMT in gastric cancer by targeting FOXQ1. Cell Physiol Biochem 36: 1382-1394, 2015. 